\title{
Reliable Slicing with Isolation in Optical Metro-Aggregation Networks
}

\author{
A. Marotta ${ }^{1}$, D. Cassioli ${ }^{1}$, M. Tornatore ${ }^{2,3}$, Y. Hirota ${ }^{4}$, Y. Awaji ${ }^{4}$, B. Mukherjee ${ }^{3}$ \\ ${ }^{1}$ University of L'Aquila, L'Aquila, Italy; ${ }^{2}$ Politecnico di Milano, Milano, Italy; \\ ${ }^{3}$ University of California, Davis, CA; ${ }^{4}$ National Institute of Information and Comm. Technology, Tokyo, Japan \\ andrea.marotta@univaq.it
}

\begin{abstract}
We discuss how different degrees of slice isolation influence resource allocation in protected optical metro-aggregation networks. The case of slice reliability with dedicated protection at lightpath is modelled and numerically evaluated. (C) 2019 The Author(s)
\end{abstract}

OCIS codes: 060.4256 Networks, network optimization; 060.4264 Networks, wavelength assignment

\section{Introduction}

The provisioning of new services with ambitious requirements has boosted the definition of the future $5 \mathrm{G}$ network architecture, where network function virtualization and service chaining are expected to enable high degree of flexibility, efficiency and reliability [1]. In 5G networks a chain of virtual links, instantiated over multiple physical links, and Virtual Network Functions (VNFs), instantiated over multiple substrate nodes, can be seen as a "network slice." Network slicing allows the composition, on demand, of virtual networks (i.e., network slices) over an infrastructure encompassing User Equipments (UEs), Radio Access Network (RAN), transport network, and core network [2].

Network slices may employ different levels of VNF isolation, that can be categorized from IO to I4, as shown in Fig. 1. In IO, concatenated VNFs with dedicated logical links constitute a "dedicated logical network", where slices experience the maximum degree of isolation. Moving from I1 to I4, the networks slices start sharing one or more VNFs, starting from the Remote Unit (RU), the Distributed Unit (DU), the Central Unit (CU) up to the Next Generation Core (NGC) element, thus decreasing the level of isolation. Fig. 1 shows the extent at which two slices share VNF with different level of isolation. The shared VNFs are referred as the "Common Segment". While it has been demonstrated, both theoretically and experimentally [3], that slicing offers high flexibility in resource management, reliability aspects of network slicing have not yet been adequately investigated. For example, among other works, reliable slicing has been investigated considering dedicated protection over elastic optical networks in [4], and considering slice recovery in 5G mobile networks in [5], but to the best of our knowledge, no study has so far evaluated the impact of different isolation levels on the resource occupation of reliable slicing.

In this study, we discuss how different degrees of isolation influence the amount of network and processing resources required for slicing in optical metro-aggregation networks. Then, we model and numerically evaluate the specific case of reliable slicing with dedicated protection against single-link failure based on a multi-layer protection approach named "Protection At Lightpath" (PAL) [6]. Our numerical results show the impact of reliable slicing with different isolation levels on resources consumption considering different strategies for VNF Isolation, network isolation, as well as for different VNF deployment scenarios (as explained more in the following Section).

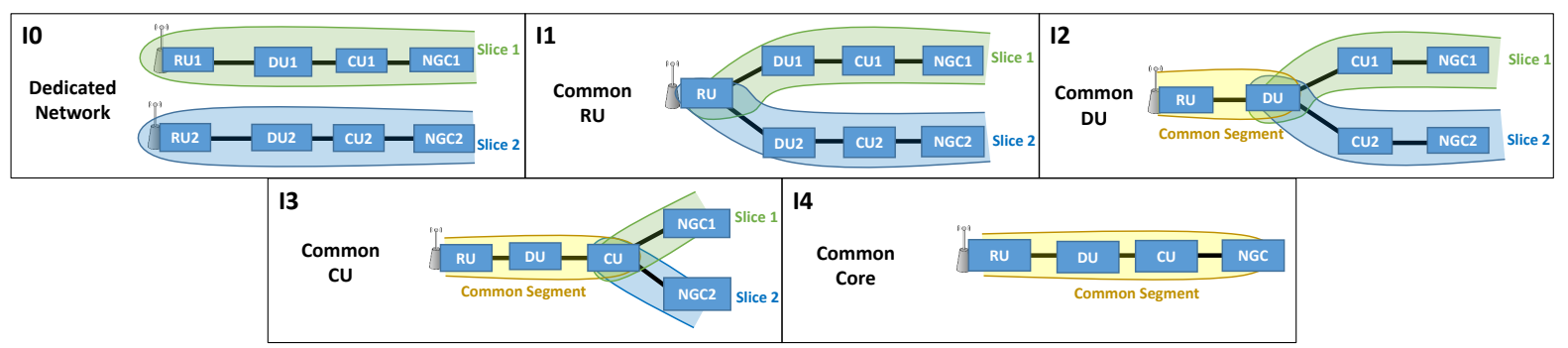

Fig. 1: Slice isolation overview

\section{Slice Isolation Overview}

Besides VNF isolation, other forms of isolation can be identified considering how slices share the resources in the networks. As shown in Fig. 2a, among this we consider the network isolation, i.e. the possibility to serve different slices over common network resources (soft isolation) or with dedicated virtual circuits (firm), lightpaths (hard), and fibers (hardest). Another important aspect is the VNF deployment strategy. We consider two VNF deployment 
strategies: i) Virtual Network Embedding (VNE), where different VNFs of the same slice must be mapped over different physical nodes; ii) Service Function Chaining (SFC), where it is allowed for different VNFs of the same slice to be co-located in the same physical node.The two different VNF deployment strategies are expected to significantly impact the number of virtual links, and consequently network resources consumption.

After having discussed various forms of slice isolation, we observe that also slice protection can be achieved through a variety of approaches, e.g., considering the entity to be protected (links, nodes, subpath, end-to-end), how backup resources are provisioned (dedicated protection, shared protection), and the layer at which the protection is implemented (physical, i.e, WDM or logical, i.e., IP). In this work we focus on the protection of the virtual links composing the slices with dedicated protection applied at lightpath layer.

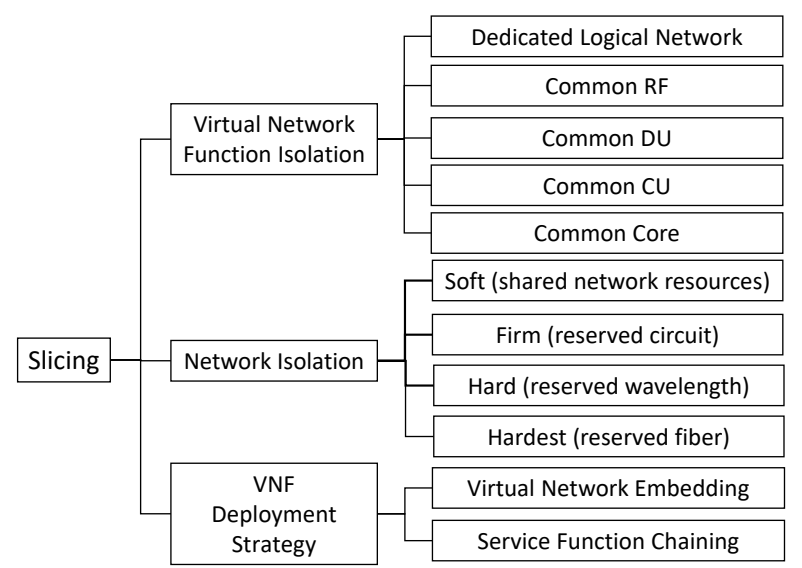

(a)

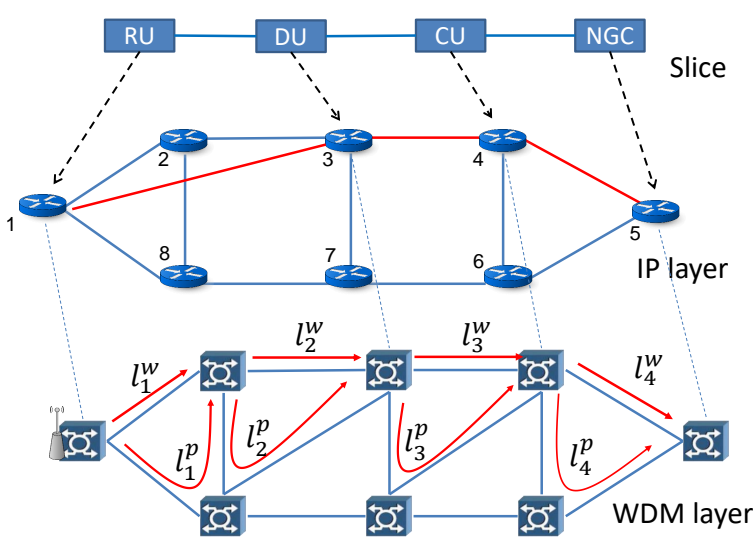

(b)

Fig. 2: (a) Slice isolation overview; (b) PAL example

\section{Reliable Slicing with Protection at Lightpath}

A viable solution to ensure slice reliability is to protect the virtual links composing the slices against link failures in the underlying physical WDM network using dedicated backup paths. We assume a slice request as represented by an antenna site and by the required wireless capacity at the antenna site. To each slice request we associate a set of virtual nodes (namely RU, DU, CU, and NGC) and virtual links (fronthaul, midhaul, and backhaul) each with specific capacity requirements. PAL provides end-to-end protection with respect to a lightpath. While traditional PAL addresses the problem of working and backup path provisioning, our problem consists in the joint optimization of: i) the mapping of virtual nodes over physical nodes in the network; ii) the mapping of the set of virtual links composing the slices each onto one or more protected lightpaths, that are defined as p-lightpaths $\left(l_{i}\right)$, consisting of pairs of working $\left(l_{i}^{w}\right)$ and backup $\left(l_{i}^{p}\right)$ lightpaths. Fig. $2 \mathrm{~b}$ shows an example of reliable slicing provisioning on a multilayer network composed of a lower WDM layer and an upper electronic IP layer. The 4 VNFs composing the slice are mapped over 4 physical nodes and the virtual links are mapped on the underlying IP and WDM layers. We assume that one virtual link represents one link at the IP layer, which in turn can be mapped over one or more $\mathrm{p}$-lightpaths. This way, a connection traversing a sequence of $\mathrm{p}$-lightpaths is able to survive to any single link failures. In the example of Fig. 2b, the virtual link between the RU and DU is mapped on the IP link $(1,3)$ which is routed through the p-lightpaths $l_{1}$ and $l_{2}$.

We formulate our problem as an Integer Linear Problem (ILP) whose mathematical description is not reported due to space limitations. A more formal description of the problem is given as follows: Given the network physical topology, the number of wavelengths, the capacity of each wavelength, the set of virtual nodes and of virtual links composing the slices, the computational capacity of each physical node decide the placement of each virtual node, the grooming, the routing and wavelength assignment of working and backup lightpaths to minimize the total wavelength channels utilization and number of active nodes such that each working lightpath has an associated link-disjoint backup lightpath; the sum of the virtual links mapped on a wavelength does not exceed the capacity of the wavelengths; the sum of computational required capacity of the virtual nodes deployed on a physical node does not exceed the computational capacity of the physical node; each virtual node is deployed on a compatible physical node; each physical node hosts only one virtual node belonging to the same slice request (in case of virtual network embedding).

\section{Results and conclusion}

We consider a metro topology organized on three hierarchical layers in which we focus on a subset of seven nodes representing Central Offices (COs). Each node is equipped with switching devices as well as computation 
infrastructures to deploy virtual functions. Access COs provide connectivity at the antenna sites and are able to host RU functions. Main COs are able to host DU and CU functionalities and provide connectivity between Access COs and the Core CO which is designed to host NGC function. Each link is equipped with one fiber per direction each supporting 40 wavelengths with $40 \mathrm{Gbit} / \mathrm{s}$ capacity. We model the computational load in terms of Processing Units (PUs) and assume required load for RU, DU, CU and NGC equal to 1PU, 0.4PU, 0.2PU, and 0.5PU respectively [7]. Switching of the traffic can be purely optical (without grooming) or opto-electronic by means of electronic switches that can rearrange input wavelength flows into output wavelengths. Slice requests are generated randomly at antenna points with capacity requirements with a normal truncated distribution with $2 \mathrm{~Gb} / \mathrm{s}$ average. System bandwidth is $100 \mathrm{MHz}$ resulting in a maximum wireless capacity of $4 \mathrm{~Gb} / \mathrm{s}$, fronthaul traffic $22 \mathrm{~Gb} / \mathrm{s}$, midhaul traffic $4,016 \mathrm{~Gb} / \mathrm{s}$ and maximum backhaul $4 \mathrm{~Gb} / \mathrm{s}$. We conducted our simulative experiment using Java-based Net2Plan environment.

Fig. 3a shows the total number of utilized wavelength channels when soft network isolation is adopted, i.e., when different slices can share wavelength channels, for both the VNE and SFC strategies. It can be noticed that in both cases, VNF isolation has no impact on optical resource utilization. This is due to the effect of grooming on network utilization. Note that higher VNF isolation has the effect of increasing the total number of virtual links, but, through grooming, different virtual links which are separated at the IP layer are routed together over common wavelengths, thus compensating the impact of VNF isolation. Fig. $3 \mathrm{~b}$ considers the hard physical isolation case with dedicated wavelengths. It can be noticed that when slices are routed over dedicated wavelengths, the VNF isolation impacts optical resources consumption, due to the different number of virtual links to be mapped that is maximum for I0 and minimum for I4. While the resource consumption for network soft isolation is significantly lower with respect to network hard isolation, it is worth to mention that the latter dramatically reduces the cost of the network equipment which are not required to support traffic grooming. The co-location of functions on the same physical node through service function chaining reduces the wavelength channels utilization since a lower number of virtual links are mapped onto the physical network. Furthermore, VNF consolidation obtained through service function chaining reduces the number of active nodes in the network at the price of increasing the average load per node, as shown in Fig. 3c. On this hand, results show that in both SFC and VNE cases, VNF isolation has the effect of increasing the average load of the nodes since it increases the total number of deployed VNFs.

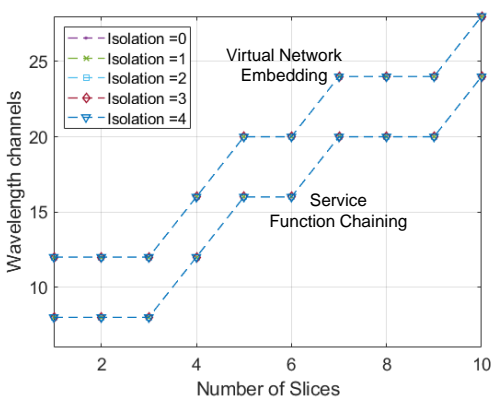

(a)

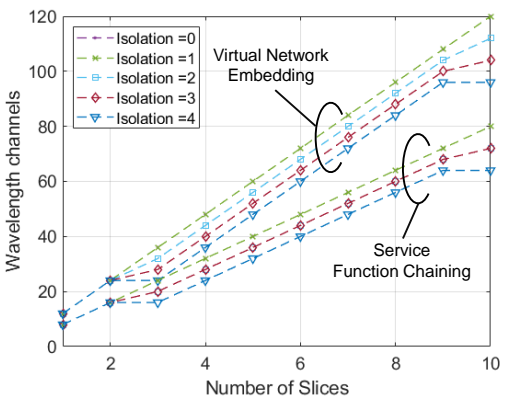

(b)

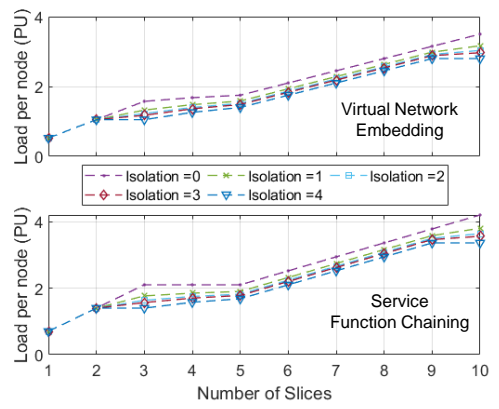

(c)

Fig. 3: Wavelength utilization for different VNF isolation degrees with network (a) soft isolation and (b) hard isolation; (c) processing load for different VNF isolation degrees and deployment strategies

\section{Acknowledgment}

This work was partially supported by the National Science Foundation Grant 1716945 and by the Italian Government under CIPE resolution no. 135 (December 21, 2012), project INnovating City Planning through Information and Communication Technologies (INCIPICT).

\section{References}

1. 5GPPP Architecture Working Group, "View on 5G Architecture," White Paper (2019).

2. NGMN Alliance, "Description of Network Slicing Concept," White Paper, v.1.0.8 (2016).

3. P. Alemany et al., "Experimental validation of network slicing management for vertical applications on multimedia real-time communications over a packet/optical network," in " 21 st International Conference on Transparent Optical Networks (ICTON)," (2019).

4. N. Shahriar et al., "Reliable Slicing of 5G Transport Networks with Dedicated Protection," (2019).

5. R. Wen et al., "On robustness of network slicing for next-generation mobile networks," IEEE Trans. on Comm. 67, 430-444 (2019).

6. C. S. Ou et al., "Traffic grooming for survivable wdm networks: dedicated protection,invited," J. Opt. Netw. 3, 50-74 (2004).

7. D. Harutyunyan et al., "Flex5g: Flexible functional split in 5g networks," IEEE Trans. on Network and Service Management 15, 961-975 (2018). 